\title{
Sildenafilo: Prevalencia y factores asociados a su consumo en estudiantes universitarios
}

\section{Sildenafil: Prevalence and Factors Associated with its Consumption in University Students}

\author{
Ledmar Jovanny Vargas Rodríguez ${ }^{1}$ Carlos Alberto Niño Avendaño ${ }^{2}$ Pedro Felipe Tristancho ${ }^{3}$ \\ ${ }^{1}$ Departamento de Epidemiología, Universidad de Boyacá, Tunja, Colombia \\ 2 Departamento de Farmacología, Escuela de Medicina, Universidad \\ Address for correspondence Carlos Alberto Niño Avendaño, MD, \\ Pedagógica y Tecnológica de Colombia (U.P.T.C), Tunja, Colombia \\ MSc, Departamento de Farmacología, Escuela de Medicina, \\ Universidad Pedagógica y Tecnológica de Colombia (U.P.T.C), \\ 3 Programa de Medicina, Universidad de Boyacá, Tunja, Colombia \\ Tunja, Colombia (e-mail: carlos.nino01@uptc.edu.co).
}

Urol Colomb 2021;30:59-65.

\section{Resumen}

Palabras clave

- adulto joven

- conducta sexual

- disfunción eréctil

- conducta social
Objetivo Determinar la prevalencia y los factores asociados al consumo de sildenafilo en estudiantes universitarios.

Métodos Se realizó un estudio observacional analítico, en estudiantes de la Facultad de Ciencias de la Salud de Instituciones de Educación Superior del municipio de Tunja. La variable dependiente fue el consumo de sildenafilo, las variables independientes fueron: edad, semestre, universidad, estrato, procedencia, pareja estable, actividad sexual actual, actividad física, inicio de las relaciones sexuales, uso de métodos de barrera, asesoría en planificación y disfunción eréctil.

Resultados La prevalencia de consumo de sildenafilo en universitarios fue del 19,25\%. Los factores que aumentan el riesgo de consumo son: tener pareja estable, actividad sexual actual, inicio de relaciones sexuales antes de los 14 años y la presencia de disfunción eréctil; mientras se disminuye dicha probabilidad en: los primeros estratos socioeconómicos, no haber escuchado sobre el medicamento, asesoría del personal de salud, conocer las indicaciones, contraindicaciones, efectividad y reacciones adversas del medicamento.

Conclusiones Una quinta parte de los universitarios usan o han usado el sildenafilo. Casi el $80 \%$ de ellos han presentado efectos adversos. El consumo frecuente de ese fármaco amerita seguir estudiando esa población universitaria, puesto que no es usual que a esa edad se presente disfunción eréctil, para conllevar a la automedicación del sildenafil por lo tanto, esa conducta debe desencadenar intervenciones desde el área de bienestar universitario, específicamente médica para que se creen estrategias de intervención que apunten a una orientación de sexualidad responsable, y prevenir su uso indiscriminado que puede llevar a riesgos demasiado graves para la salud tanto física como mental. received

May 1, 2020

accepted

July 20, 2020

published online

January 13, 2021
DOI https://doi.org/

10.1055/s-0040-1716680.

ISSN 0120-789X.

e ISSN 2027-0119.
(C) 2021. Sociedad Colombiana de Urología. All rights reserved. This is an open access article published by Thieme under the terms of the Creative Commons Attribution-NonDerivative-NonCommercial-License, permitting copying and reproduction so long as the original work is given appropriate credit. Contents may not be used for commercial purposes, or adapted, remixed, transformed or built upon. (https://creativecommons.org/ licenses/by-nc-nd/4.0/)

Thieme Revinter Publicações Ltda., Rua do Matoso 170, Rio de Janeiro, RJ, CEP 20270-135, Brazil 


\begin{abstract}
Keywords

- young adult

- sexual conduct

- erectile dysfunction

- social conduct

Objective To determine the prevalence and factors associated with the consumption of sildenafil in university students.

Methods An analytical observational study was carried out in students of the Faculty of Health Sciences of Institutions of Higher Education in the municipality of Tunja. The dependent variable was the consumption of sildenafil, the independent variables were: age, semester, university, stratum, provenance, stable partner, current sexual activity, physical activity, initiation of sexual relations, use of barrier methods, planning advice and erectile dysfunction.

Results The prevalence of sildenafil consumption in university students was $19.25 \%$. The factors increase the risk of consumption are: having a stable partner, current sexual activity, initiation of sexual relations before the age of 14 and the presence of erectile dysfunction; while this probability is decreased in: the first socioeconomic strata, not having heard about the drug, advice from health personnel, knowing the indications, contraindications, effectiveness and adverse reactions of the drug. Conclusions A fifth of university students use or have used sildenafil. Almost $80 \%$ of them have presented adverse effects. Frequent consumption of this drug merits further study in this university population, since it is unusual for erectile dysfunction to occur at this age, leading to self-medication of sildenafil, therefore this behavior should trigger interventions from the university wellness area. , specifically medical, so that intervention strategies are created that aim to give a responsible orientation of sexuality, and prevent its indiscriminate use that can lead to too serious risks for both physical and mental health.
\end{abstract}

\section{Introducción}

La dificultad en la respuesta sexual representa una preocupación y sufrimiento para las personas que las padecen y sus parejas. En el caso de la disfunción eréctil (DE), la cual compromete de manera significativa el desempeño sexual masculino, puede llevar a situaciones personales difíciles, comprometer la pareja y la autoestima de quienes la sufren. ${ }^{1}$ La disfunción eréctil (DE) es la incapacidad de lograr o mantener una erección del pene para la penetración vaginal y el desempeño sexual satisfactorio, y se le considera el segundo problema más frecuente de disfunción sexual en los hombres, después de la eyaculación precoz, con una prevalencia aproximada del $30 \%{ }^{2}$

En cuanto a la etiología de la disfunción eréctil, tenemos desde la psicológica como la ansiedad de ejecución, descrita por Masters y Johnson, ${ }^{3}$ que presentan determinados hombres después de haber experimentado algún fracaso de la erección, hasta la relacionada con la pareja, ya sea por problemas de diálogo o comunicación, por falta de intimidad o de confianza o por conflictos de poder ${ }^{4}$. Igualmente diversos estudios sugieren que los hombres con factores de riesgo cardiovascular como obesidad, diabetes mellitus, hipertensión, dislipidemia tienen un riesgo de padecer (DE) significativamente más alto ${ }^{5}$; es así que la aterogénesis, con la disfunción endotelial, ha sido relacionada con el síndrome metabólico y es la base fisiopatológica de la enfermedad cardiovascular.
Caracterizada por una deficiente síntesis, liberación y actividad principalmente del óxido nítrico por el endotelio vascular, ${ }^{6}$ la disfunción endotelial también se traduce en un deterioro de la capacidad de mantener la erección del pene, que culmina en la (DE). ${ }^{7,8}$ Además de otras causas de origen neurológicas, anatómicas y endocrinológicas. ${ }^{9}$

En el caso de los pacientes jóvenes, se establece que no es frecuente la disfunción eréctil, ya que ella aumenta con la edad, es así que se estima que del 5\% al $47 \%$ de la población adulta, presenta o manifiesta (DE) moderada a completa. Esa proporción aumenta en relación directa con la edad. ${ }^{10}$

Referente al tratamiento de la disfunción eréctil, hay alternativas farmacológicas para su tratamiento, es el caso de los inhibidores de la PDE5, los cuales son antagonistas competitivos de la acción de la PDE5 que inducen aumento intracelular de los niveles de GMP cíclico en el músculo cavernoso, lo que conlleva la relajación del músculo liso y la erección en respuesta al estímulo sexual. ${ }^{11}$

El consumo de esos medicamentos debe estudiarse adecuadamente entre la población joven, para hacer intervenciones encaminadas a una sexualidad responsable, y prevenir la automedicación, ${ }^{12}$ ya que hay estudios que evidencian una prevalencia importante, donde se detectó que el $35 \%$ de quienes consumen sidenafilo son hombres entre 25 y 35 años. $^{13}$ 
El objetivo del estudio fue determinar la prevalencia y los factores asociados al consumo de sildenafilo en estudiantes universitarios.

\section{Métodos}

Se realizó un estudio observacional analítico, efectuado entre el 15 de enero y el 10 de Marzo del 2020 en estudiantes de Instituciones de Educación Superior (IES) del municipio de Tunja, donde se incluyeron a varones entre los 18 y los 37 años de edad, excluyendo a todos los participantes que diligenciaran la encuesta de manera inadecuada o que no aceptaran la participación en el estudio.

El tamaño de la muestra se calculó con el programa Epi Info versión 7.2. Se tomó como base la población total de hombres registrados en el sistema de admisiones de las facultades de Ciencias de la Salud de dos IES, que cumplieran los criterios de selección (n: 4215). La proporción que se esperó encontrar de uso de sildenafilo fue del $73,2 \%,{ }^{14}$ se estimó intervalos de confianza (IC) del 95\% y un error admisible del 5\%, permitiendo pérdidas del 20\%; lo anterior arrojó la cantidad de 322 estudiantes a encuestar. Los participantes fueron seleccionados mediante un muestreo probabilístico estratificado por afijación igual.

La variable dependiente fue el consumo de sildenafilo (citrato de sildenafilo) en los últimos 12 meses y las variables independientes estudiadas fueron: edad, semestre que se encuentra cursando, tipo de universidad (privada/publica) estrato socioeconómico, procedencia, pareja estable, actividad sexual actual, realización de actividad física, edad de inicio de las relaciones sexuales, uso de métodos de barrera durante las relaciones sexuales, asesoría en planificación por personal de la salud, presencia de disfunción eréctil (incapacidad repetida de alcanzar y sostener una erección suficientemente firme para mantener una relación sexual), conocimiento acerca de la existencia del medicamento y los efectos adversos que genera.

Adicionalmente, los estudiantes que refirieron consumir ese medicamento, se les indagó acerca de la frecuencia de uso (en cada relación sexual, una vez al día, una vez a la semana, una vez al mes, una vez al año, uso anecdótico (solo en una ocasión para saber que sucedía) y los efectos adversos que presentaron.

La base de datos fue registrada en Excel versión 2013 y se analizó en el paquete estadístico SPSS versión 22 . El análisis univariado se realizó por medio de un estadístico descriptivo a cada grupo (según hubiesen consumido o no sildenafilo), determinando frecuencias absolutas y relativas en las variables categóricas, en el caso de las variables cuantitativas se calcularon las medidas de tendencia central (media, mediana) y medidas de dispersión (desviación estándar y rango intercuartil). En el análisis bivariado para determinar las posibles asociaciones entre variables categóricas, se realizaron tablas tetracoricas, con medición de Chi cuadrado de Pearson y Odds ratio (OR) con su respectivos IC al 95\%
( $<1$ como factor protector, $>1$ como factor de riesgo y 1 sin asociación). El análisis multivariado se realizó mediante una regresión logística binaria, tomando como base aquellas variables que tuvieron asociación con el uso o consumo del citrato de sildenafilo.

El estudio se desarrolló de acuerdo con las normas establecidas en la Resolución 8430 de 1993 del Ministerio de Salud. De acuerdo con su artículo 11 este estudio se clasifica como una investigación "sin riesgo", ya que no se realizó ninguna intervención o modificación intencionada de las variables biológicas, fisiológicas, sicológicas o sociales de los individuos que participan en el estudio. La participación fue voluntaria y quienes aceptaron formar parte del estudio firmaron un consentimiento informado. Los datos fueron manejados con estricta confidencialidad y anonimato, sin realizarse análisis individuales; el formato de valoración estaba numerado con un código el cual solamente fue útil para el proceso de tabulación, y estaba separado del registro de consentimiento informado.

Los sesgos que se pueden presentar en la presente investigación, son el de selección, pero se describieron los criterios de inclusión y exclusión con el fin de seleccionarlos de manera adecuada, además se realizó un proceso de aleatorización para que todos tuvieran la misma posibilidad de ingresar al estudio. El segundo sesgo que se puede presentar es el de información, puesto que al tratarse de encuestas se pueden presentar problemas con los datos recolectados, por lo que los investigadores revisaban las encuestas posterior a su entrega con el fin de verificar los datos completos, adicionalmente, se calculó un porcentaje de pérdida en el tamaño de la muestra, con el fin de evitar problemas con el número de encuestados.

\section{Resultados}

\section{Selección de los participantes}

Según las bases de datos de las IES, se contaba con un total del 4215 estudiantes, a los que se les realizó un muestreo probabilístico estratificado por afijación igual para la selección de los participantes, dentro de los que se seleccionaron 322 estudiantes hombres, divididos en 161 pertenecientes a la universidad pública y 161 de la universidad privada, a los cuales se les aplicó las encuestas.

\section{Prevalencia y frecuencia de consumo}

De los 322 participantes, el 19,25\% (n: 62) han usado el citrato de sildenafilo en algún momento de su vida. Dentro de la frecuencia de uso se encontró que el $41,94 \%$ ha sido de uso anecdótico (una sola vez en la vida, el 22,58\% lo usan una vez al año, el 19,35\% lo usan en cada relación sexual y el 16,13\% lo usan una vez al mes.

\section{Efectos adversos}

Del total de participantes, el 20,9\% refieren que no han presentado ningún efecto adverso secundario al uso del sildenafilo. En la - Tabla 1, se presentan los efectos adversos que presentaron. 
62 Sildenafilo: Prevalencia y factores asociados a su consumo en estudiantes universitarios Vargas-Rodriguez y col.

Tabla 1 Efectos adversos referidos

\begin{tabular}{|l|l|l|}
\hline Efectos secundarios & N & $\%$ \\
\hline Ninguno & 13 & 20,97 \\
\hline Cefalea & 38 & 61,29 \\
\hline Dolor torácico & 15 & 24,19 \\
\hline Mareo & 24 & 38,71 \\
\hline Visión borrosa & 10 & 16,13 \\
\hline Enrojecimiento ocular & 8 & 12,90 \\
\hline Insomnio & 7 & 11,29 \\
\hline Taponamiento nasal & 8 & 12,90 \\
\hline Edema facial & 3 & 4,84 \\
\hline Palpitaciones & 2 & 3,23 \\
\hline
\end{tabular}

\section{Características sociodemográficas}

La mayor parte de los encuestados tenían entre 16 a 21 años de edad, con estrato socioeconómico entre 1 a 3 , en el momento sin pareja estable y de procedencia urbana. Las características de los participantes se muestran en la -Tabla 2.

\section{Factores asociados al consumo de sildenafilo}

Se determinó que los factores que aumentan el riesgo de consumo de citrato de sildenafilo son: tener pareja estable, actividad sexual actual, inicio de relaciones sexuales antes de los 14 años de edad y la presencia de disfunción eréctil; mientras que los que disminuyen la probabilidad de consumo son: estrato socioeconómico 1 a 3, no conocer (o haber escuchado) sobre el medicamento, recibir asesoría del personal de salud, conocer los efectos y reacciones adversas del medicamento ( - Tabla $\mathbf{3}$ ).

Tabla 2 Características de los participantes

\begin{tabular}{|c|c|c|c|c|c|}
\hline \multicolumn{2}{|l|}{ Variables } & \multicolumn{2}{|c|}{ Uso de sildenafilo } & \multirow[t]{2}{*}{ Total (n:322) } & \multirow[b]{2}{*}{ Valor $\mathrm{p}$ (Chi2) } \\
\hline & & Si $(n: 62)$ & No $(n: 260)$ & & \\
\hline \multirow[t]{2}{*}{ Universidad } & Privada & 37 & 124 & 161 & \multirow[t]{2}{*}{0,901} \\
\hline & Publica & 25 & 136 & 161 & \\
\hline \multirow[t]{4}{*}{ Grupos de edad } & 16 a 21 años & 41 & 187 & 228 & 0,367 \\
\hline & 22 a 26 años & 15 & 65 & 80 & 0,895 \\
\hline & 27 a 31 años & 3 & 5 & 8 & 0,185 \\
\hline & 32 a 36 años & 3 & 3 & 6 & 0,117 \\
\hline \multirow[t]{2}{*}{ Estrato socioeconómico } & 1 a 3 & 34 & 177 & 211 & \multirow[t]{2}{*}{0,490} \\
\hline & 4 a 6 & 28 & 83 & 111 & \\
\hline \multirow[t]{2}{*}{ Pareja estable: } & Sí & 15 & 25 & 40 & \multirow[t]{2}{*}{0,002} \\
\hline & No & 47 & 235 & 282 & \\
\hline \multirow[t]{2}{*}{ Actividad sexual actual: } & Sí & 55 & 185 & 240 & \multirow[t]{2}{*}{0,004} \\
\hline & No & 7 & 75 & 82 & \\
\hline \multirow[t]{2}{*}{ Procedencia } & Urbana & 46 & 218 & 264 & \multirow[t]{2}{*}{0,076} \\
\hline & Rural & 16 & 42 & 58 & \\
\hline \multirow[t]{3}{*}{ Semestre } & $<4$ semestre & 18 & 77 & 95 & 0,928 \\
\hline & 5 a 8 semestre & 31 & 158 & 189 & 0,122 \\
\hline & 9 semestre en adelante & 13 & 25 & 38 & 0,013 \\
\hline \multirow[t]{2}{*}{ ¿Realiza actividad física? } & Sí & 54 & 201 & 255 & \multirow[t]{2}{*}{0,088} \\
\hline & No & 8 & 59 & 67 & \\
\hline \multirow{3}{*}{$\begin{array}{l}\text { Edad de inicio de las } \\
\text { relaciones sexuales }\end{array}$} & Antes de los 14 años & 17 & 43 & 60 & 0,048 \\
\hline & Entre 15 a 18 años & 36 & 176 & 212 & 0,151 \\
\hline & Después de los 19 años & 9 & 41 & 50 & 0,807 \\
\hline \multirow{2}{*}{$\begin{array}{l}\text { ¿Utiliza condón en las } \\
\text { relaciones sexuales? }\end{array}$} & Sí & 43 & 194 & 237 & \multirow[t]{2}{*}{0,398} \\
\hline & No & 19 & 66 & 85 & \\
\hline \multirow{2}{*}{$\begin{array}{l}\text { ¿Usted conoce el medicamento } \\
\text { conocido como sildenafil (viagra)? }\end{array}$} & Sí & 62 & 173 & 235 & \multirow[t]{2}{*}{0,001} \\
\hline & No & 0 & 87 & 87 & \\
\hline \multirow{2}{*}{$\begin{array}{l}\text { ¿Recibió asesoría o información } \\
\text { de personal de la salud? }\end{array}$} & Sí & 17 & 2 & 19 & \multirow[t]{2}{*}{0,002} \\
\hline & No & 45 & 258 & 303 & \\
\hline \multirow{2}{*}{$\begin{array}{l}\text { ¿El uso de sildenafil, está asociado } \\
\text { a disfunción eréctil? }\end{array}$} & Sí & 45 & 34 & 79 & \multirow[t]{2}{*}{0,000} \\
\hline & No & 17 & 226 & 243 & \\
\hline \multirow{2}{*}{$\begin{array}{l}\text { ¿Usted conoce los efectos } \\
\text { secundarios del sildenafil? }\end{array}$} & Sí & 41 & 62 & 103 & \multirow[t]{2}{*}{0,000} \\
\hline & No & 21 & 198 & 219 & \\
\hline
\end{tabular}


Tabla 3 Factores asociados al consumo de sildenafilo

\begin{tabular}{|c|c|c|c|c|c|c|c|}
\hline \multicolumn{2}{|l|}{ Variables } & \multirow{2}{*}{$\begin{array}{l}\text { OR crudo } \\
1,480\end{array}$} & \multicolumn{2}{|c|}{ IC 95\% } & \multirow{2}{*}{$\begin{array}{l}\text { OR ajustado } \\
\text { N/A }\end{array}$} & \multicolumn{2}{|c|}{ IC 95\% } \\
\hline Universidad & Privada & & 0,936 & 2,340 & & $N / A$ & $N / A$ \\
\hline \multirow[t]{4}{*}{ Grupos de edad } & 16 a 21 años & 0,805 & 0,504 & 1,285 & $\mathrm{~N} / \mathrm{A}$ & $\mathrm{N} / \mathrm{A}$ & $N / A$ \\
\hline & 22 a 26 años & 0,965 & 0,572 & 1,629 & $\mathrm{~N} / \mathrm{A}$ & $\mathrm{N} / \mathrm{A}$ & $\mathrm{N} / \mathrm{A}$ \\
\hline & 27 a 31 años & 1,996 & 0,792 & 5,026 & $\mathrm{~N} / \mathrm{A}$ & $\mathrm{N} / \mathrm{A}$ & $\mathrm{N} / \mathrm{A}$ \\
\hline & 32 a 36 años & 2,650 & 0,969 & 7,248 & $\mathrm{~N} / \mathrm{A}$ & $\mathrm{N} / \mathrm{A}$ & $\mathrm{N} / \mathrm{A}$ \\
\hline \multirow[t]{2}{*}{ Estrato socioeconómico } & 1 a 3 & 0,639 & 0,410 & 0,996 & 0,721 & 0,352 & 0,882 \\
\hline & 4 a 6 & 1,122 & 0,992 & 1,269 & $\mathrm{~N} / \mathrm{A}$ & $\mathrm{N} / \mathrm{A}$ & $\mathrm{N} / \mathrm{A}$ \\
\hline \multirow[t]{2}{*}{ Pareja estable } & $\mathrm{Si}$ & 2,250 & 1,396 & 3,628 & 3,572 & 1,152 & 5,876 \\
\hline & No & 0,750 & 0,587 & 0,959 & 0,842 & 0,675 & 0,965 \\
\hline \multirow[t]{2}{*}{ Actividad sexual actual } & $\mathrm{Si}$ & 2,685 & 1,274 & 5,658 & 3,648 & 2,156 & 5,374 \\
\hline & No & 0,843 & 0,766 & 0,927 & 0,846 & 0,673 & 0,942 \\
\hline \multirow[t]{2}{*}{ Procedencia } & Urbana & 0,632 & 0,386 & 1,034 & $\mathrm{~N} / \mathrm{A}$ & $N / A$ & $N / A$ \\
\hline & Rural & 1,140 & 0,964 & 1,349 & $\mathrm{~N} / \mathrm{A}$ & $\mathrm{N} / \mathrm{A}$ & $\mathrm{N} / \mathrm{A}$ \\
\hline \multirow[t]{3}{*}{ Semestre cursado } & 4 o menor & 0,978 & 0,597 & 1,601 & $\mathrm{~N} / \mathrm{A}$ & $\mathrm{N} / \mathrm{A}$ & $N / A$ \\
\hline & 5 a $8^{\circ}$ & 0,704 & 0,451 & 1,099 & $\mathrm{~N} / \mathrm{A}$ & $\mathrm{N} / \mathrm{A}$ & $\mathrm{N} / \mathrm{A}$ \\
\hline & $9^{\circ}$ en adelante & 1,983 & 1,192 & 3,299 & 1,275 & 0,892 & 0,864 \\
\hline \multirow[t]{2}{*}{ ¿Realiza actividad física? } & Sí & 1,774 & 0,888 & 3,543 & $\mathrm{~N} / \mathrm{A}$ & $\mathrm{N} / \mathrm{A}$ & $\mathrm{N} / \mathrm{A}$ \\
\hline & No & 0,895 & 0,803 & 1,001 & $\mathrm{~N} / \mathrm{A}$ & $\mathrm{N} / \mathrm{A}$ & $\mathrm{N} / \mathrm{A}$ \\
\hline \multirow{3}{*}{$\begin{array}{l}\text { Edad de inicio de las } \\
\text { relaciones sexuales }\end{array}$} & Antes de los 14 años & 1,650 & 1,184 & 2,672 & 2,135 & 1,349 & 4,21 \\
\hline & 15 a 18 años & 0,718 & 0,459 & 1,125 & $\mathrm{~N} / \mathrm{A}$ & $\mathrm{N} / \mathrm{A}$ & $\mathrm{N} / \mathrm{A}$ \\
\hline & 19 años en adelante & 0,924 & 0,488 & 1,750 & $\mathrm{~N} / \mathrm{A}$ & $\mathrm{N} / \mathrm{A}$ & $\mathrm{N} / \mathrm{A}$ \\
\hline \multirow{2}{*}{$\begin{array}{l}\text { Uso de métodos de barrera } \\
\text { en las relaciones sexuales }\end{array}$} & Sí & 0,812 & 0,502 & 1,311 & $\mathrm{~N} / \mathrm{A}$ & $\mathrm{N} / \mathrm{A}$ & $\mathrm{N} / \mathrm{A}$ \\
\hline & No & 1,054 & 0,927 & 1,199 & $\mathrm{~N} / \mathrm{A}$ & $\mathrm{N} / \mathrm{A}$ & $N / A$ \\
\hline $\begin{array}{l}\text { ¿Usted conoce el medicamento } \\
\text { conocido como sildenafil? }\end{array}$ & No & 0,736 & 0,682 & 0,795 & 0,317 & 0,015 & 0,642 \\
\hline \multirow{2}{*}{$\begin{array}{l}\text { ¿Recibió asesoría de } \\
\text { personal de la salud? }\end{array}$} & Sí & 0,124 & 0,033 & 0,459 & 0,248 & 0,021 & 0,512 \\
\hline & No & 6,025 & 4,416 & 8,219 & 4,753 & 3,951 & 5,486 \\
\hline \multirow{2}{*}{$\begin{array}{l}\text { ¿Usted conoce los efectos } \\
\text { secundarios del sildenafil? }\end{array}$} & Sí & 0,666 & 0,566 & 0,784 & 0,152 & 0,082 & 0,218 \\
\hline & No & 4,151 & 2,592 & 6,648 & 1,895 & 1,124 & 2,341 \\
\hline \multirow{2}{*}{$\begin{array}{l}\text { Uso del sildenafilo por } \\
\text { disfunción eréctil }\end{array}$} & Sí & 8,142 & 4,954 & 13,383 & 9,572 & 5,876 & 12,561 \\
\hline & No & 0,463 & 0,358 & 0,598 & 0,324 & 0,247 & 0,643 \\
\hline
\end{tabular}

\section{Discusión}

Los estudiantes universitarios son un sector de la población que debido a su periodo de transición entre la adolescencia y la adultez se ha catalogado como un momento de vivencias de alto riesgo, donde las influencias socioculturales y sexuales, ${ }^{15,16}$ les infunde la necesidad de mostrar una virilidad basada en la capacidad de responder en el acto sexual, lo cual puede conducir al consumo de sildenafil. ${ }^{17,18}$

La prevalencia de consumo hallada en población universitaria fue del $19,25 \%$, donde el $41,94 \%$ lo habían usado una sola vez en la vida, el $22,58 \%$ lo usan una vez al año, el 19,35\% lo usan en cada relación sexual y el 16,13\% lo usan una vez al mes. Cortez y col., ${ }^{14}$ reportó que el 73,2\% de las personas entre los 16 y los 65 años, han consumido ese medicamento alguna vez en su vida Por otro lado, Mussachio y col., ${ }^{15}$ reportó que el $6 \%$ de los varones en edades entre los 18 a 25 años han consumido alguna vez ese medicamento, y finalmente, Martínez y col., ${ }^{12}$ encontró una prevalencia de consumo en estudiantes universitarios del 7,56\%, lo cual nos hace pensar que el hallazgo de esta investigación es relativamente alto al compararse con poblaciones de características similares en edad.

Los efectos adversos más frecuentes fueron cefalea, mareos y dolor torácico, resultado dispar con el estudio realizado en España 2006 donde fueron dispepsia y cefalea. $^{19}$ El uso de viagra se ha asociado con relaciones sexuales de riesgo (mayor número de parejas sexuales, tasas más altas de infecciones de transmisión sexual y relaciones sexuales sin protección con parejas VIH positivas). ${ }^{20,21}$ 
Similar a lo reportado por Martínez y col., ${ }^{12}$ los varones que presentan disfunción eréctil (OR 21,67 IC95\% 6,2774,89) y el bajo nivel de conocimiento sobre el medicamento (OR 4,21 IC95\% 1,63- 11,3), aumenta la probabilidad de su consumo. Sin embargo, difiere de variables como el inicio de relaciones sexuales antes de los 14 años de edad (OR 0,39 (0,31-1,98), que en el presente estudio se presentó como un factor de riesgo.

Claramente, la población que presenta disfunción eréctil, tiene mayor probabilidad de consumir ese fármaco, puesto que ha mostrado efectos favorecedores, disminuyendo el riesgo de eyaculación prematura y aumento del período coital en 15 minutos para el $93 \%$ de las personas que lo usan. 22,23

La ocupación de las personas es un factor importante para el momento de la automedicación, donde la población de estudiantes de universitarios es más propenso a ese tipo de prácticas (57\%), sin embargo, eso depende de los ingresos económicos que tienen, por lo cual se asume que aquellas personas de mayor nivel socioeconómico son más propensos a automedicarse. ${ }^{24}$ En la presente investigación, se reportó que las personas de estratos 1 a 3 tienen menos riesgo de consumo de sildenafilo, sin embargo, difiero de lo encontrado por Peñuela y col., ${ }^{25}$ donde refiere que las personas de estratos 1 a 3 , tienen mayor riesgo de automedicarse (OR 1,83 (1,19-2,80). Y esa diferencia se puede deber a que en él se estudió la automedicación de otros medicamentos relacionados con cuadros como dolor, infección o gripes.

Las personas que recibieron asesoría en planificación familiar por parte del personal de la salud, tienen menos probabilidad de consumir ese medicamento Según Leape y col., ${ }^{26}$ un cuarto de la población que se automedica, no tiene los conocimientos suficientes ni se informan con el personal idóneo sobre los fármacos utilizados y su efectos adversos, lo cual puede generar lesiones e incluso discapacidad. ${ }^{26,27}$ Motivo por el cual se aconseja una adecuada asesoría a la población con el fin de conocer las indicaciones, contraindicaciones y reacciones adversas de los medicamentos, para evitar problemas de salud relacionados con el uso inadecuado de los medicamentos.

El consumo frecuente de ese fármaco amerita seguir estudiando esa población universitaria, puesto que no es usual que a esa edad se presente disfunción eréctil, para conllevar a la automedicación del sildenafil, por lo tanto esa conducta debe desencadenar intervenciones desde el área de bienestar universitario, específicamente médica para que se creen estrategias de intervención que apunten a dar una orientación de sexualidad responsable, y prevenir su uso indiscriminado que puede llevar a riesgos demasiado graves para la salud tanto física como mental.

Limitaciones y fortalezas: Las principales limitaciones del presente estudio están relacionadas con la información proporcionada por los encuestados, puesto que se corre el riesgo de sesgo en la memoria o sesgo por obsequiedad por parte de los participantes. Adicionalmente, es difícil evaluar la temporalidad de exposición. Sin embargo, es un estudio con importantes fortalezas, dentro de las que destacan el tamaño de muestra apropiado, junto con un análisis estadístico que permite evaluar la causalidad y los hallazgos que son de suma importancia para la población.

\section{Conclusiones}

La prevalencia de consumo de sildenafilo en esa población fue del 19,25\%. Los factores que aumentan el riesgo de consumo de citrato de sildenafilo son: tener pareja estable, actividad sexual actual, inicio de relaciones sexuales antes de los 14 años de edad y la presencia de disfunción eréctil; mientras que los que disminuyen la probabilidad de consumo son: Estrato socioeconómico 1 a 3, no conocer (o haber escuchado) sobre el medicamento, por lo cual es importante que los estudiantes reciban asesoría del personal de salud, con el propósito de conocer las indicaciones, contraindicaciones, efectividad $\mathrm{y}$ reacciones adversas del medicamento.

\section{Conflicto de Intereses}

Los autores declaran no tener ningún conflicto de intereses.

\section{Agradecimientos}

Un especial agradecimiento a los participantes del estudio por su interés en la investigación y valioso aporte generado con la información aportada.

\section{Referencias}

1 Rodríguez Rodríguez Teresa. Disfunción sexual eréctil: ¿apenas una dificultad de los órganos sexuales? Rev. Finlay 2017;7(03):225-228. Disponible en: http://scielo.sld.cu/scielo.php?script=sci_arttext\& pid=S2221-24342017000300010\&lng=es

2 García HA, Ocampo GM. Un nuevo enfoque terapéutico para la disfunción eréctil: las ondas de choque de baja intensidad. Rev Iatreia 2011;28(03. Disponible en: https://aprendeenlinea.udea. edu.co/revistas/inde x.php/iatreia/article/view/

3 Masters WH, Johnson VE. Human Sexual Inadequacy. Boston: Little Brown; 1970. Disponible en: https://onlinelibrary. wiley.com/doi/abs/10.1002/tera.1420040411

4 Monseny JM. Disfunción eréctil de origen psicogeno. Arch Esp Urol 2010;63(08):599-602

5 Bansal TC, Guay AT, Jacobson J, Woods BO, Nesto RW. Incidence of metabolic syndrome and insulin resistance in a population with organic erectile dysfunction. J Sex Med 2005;2(01):96-103

6 Bonetti PO, Lerman LO, Lerman A. Endothelial dysfunction: a marker of atherosclerotic risk. Arterioscler Thromb Vasc Biol 2003;23(02):168-175

7 Sáenz de Tejada I, Angulo J, Cellek S, et al. Physiology of erectile function. J Sex Med 2004;1(03):254-265

8 Burnett AL. Nitric oxide in the penis: physiology and pathology. J Urol 1997;157(01):320-324

9 Correa Jáuregui Manuel. Los inhibidores de las fosfodiesterasas en el tratamiento de la disfunción sexual eréctil. Rev Cuba Med Mil 2010;39(3-4):255-264. Disponible en: http://scielo.sld.cu/scielo. php?script=sci_arttext $\&$ pid=S013865572010000300010\&lng=es

10 Lue T, Basson R, Rosen R, Guiliano F, Khoury S. Sexual Medicine: Sexual Dysfunctions in Men and Women. ParisHealth Productions2004. Disponible en: https://www.ncbi.nlm.nih.gov/ pmc/articles/PMC557105/

11 María del Pilar C, Villarraga Á, Silva Herrera JD, Miguel J, Juan Fernando U, Daniel M. Guía de disfunción eréctil. Sociedad Colombiana de Urología. Revista Urología Colombiana 2015; 
XXIV(03):185.e2-185.e22. Recuperado de: https://www.redalyc. org/articulo.oa?id=149143142010

12 Torres JM, Duarte-Corredor IK, García-Vásquez GJ,Jáuregui-Rodríguez GD. Prevalencia y factores asociados al consumo de citrato de sildenafil en estudiantes universitarios colombianos, durante el primer semestre 2013. Duazary 2015;12(02):118-124. Recuperado de: https://www.redalyc.org/articulo.oa?id=512156300006

13 Rovira S. Paula, La Viagra nuestra de cada día. Consumo recreacional y angustias masculinas con respecto a su potencia eréctil. Sex Salud Soc (Rio J) 2014;(18):140-160. Recuperado de: https://www. redalyc.org/articulo.oa?id=293332699006 [Internet]

14 Cortez E, Marín M en la investigación. Estudio de la prevalencia del consumo de sildenafilo en varones de 15 a 65 años de edad de la ciudad de Cajamarca, 2016.[tesis]. Cajamarca-Perú Servicio de Publicaciones e intercambio Científico. Universidad Privada Antonio Guillermo Urrelo; 2016

15 Musacchio NS, Hartrich M, Garofalo R. Erectile dysfunction and viagra use: what's up with college-age males? J Adolesc Health 2006;39(03):452-454

16 Furstenberg FF. The sociology of adolescence and youth in the 1990s: A critical commentary. J Marriage Fam 2000;62(04): 896-910. Disponible en: https://onlinelibrary.wiley.com/doi/ abs/10.1111/j.1741-3737.2000.00896.x

17 Bynner J. Rethinking the youth phase of the life-course: The case for emerging adulthood? J Youth Stud 2005;8(04):367-384. Disponible en: https://www.tandfonline.com/doi/abs/10.1080/ 13676260500431628

18 Mennickent S, Zurita L, Vega M, Villegas G. Características del uso de Sildenafil en una muestra de pacientes a nivel de farmacias comunitarias de Concepción, Chile. Rev Med Chil 2005;133(06): 667-673

19 Rodríguez Vela L, Lledó García E, Rajmil O, Mo D, Cassinello A, Casariego J. Preferencia de tadalafilo vs sildenafilo en pacientes españoles con disfunción eréctil: resultados procedentes de un estudio multicéntrico internacional. Actas Urol Esp 2006;30(01):67-79

$20 \mathrm{Kim}$ AA, Kent CK, Klausner JD. Increased risk of HIV and sexually transmitted disease transmission among gay or bisexual men who use Viagra, San Francisco 2000-2001. AIDS 2002;16(10): $1425-1428$

21 Chu PL, McFarland W, Gibson S, et al. Viagra use in a communityrecruited sample of men who have sex with men, San Francisco. J Acquir Immune Defic Syndr 2003;33(02):191-193

22 Aversa A, Mazzilli F, Rossi T, Delfino M, Isidori AM, Fabbri A. Effects of sildenafil (Viagra) administration on seminal parameters and post-ejaculatory refractory time in normal males. Hum Reprod 2000;15(01):131-134

23 Abdel-Hamid IA, El Naggar EA, El Gilany AH. Assessment of as needed use of pharmacotherapy and the pause-squeeze technique in premature ejaculation. Int J Impot Res 2001;13 (01):41-45

24 Chávez M, María L, Yenny ETB. Influencia de los factores socioeconómicos y culturales en la automedicación en usuarios de 18-64 años de edad que acuden a los establecimientos farmacéuticos en el distrito de Cajamarca 2016. 2017. Tesis de grado. Revisada el 27 de Junio del 2020. Disponible en http:// repositorio.upagu.edu.pe/handle/UPAGU/456

25 Peñuela M, de la Espriella A, Escobar E, et al. Factores socioeconómicos y culturales asociados a la autoformulación en expendios de medicamentos en la ciudad de Barranquilla. Salud uninorte. 2002:16. Disponible en: http://rcientificas.uninorte. edu.co/index.php/salud/article/viewArticle/4178/5599

26 Leape LL, Bates DW, Cullen DJ, et al; ADE Prevention Study Group. Systems analysis of adverse drug events. JAMA 1995;274(01):35-43

27 Bates DW, et al. The costs of adverse drug events in hospitalised patients. Advers a Drug Events Prevention Study Group. JAMA 1997;277(04):307-311 